\title{
One-dimensional backreacting holographic superconductors with exponential nonlinear electrodynamics
}

\author{
B. Binaei Ghotbabadi ${ }^{1}$, M. Kord Zangeneh ${ }^{2,3, a}$, A. Sheykhi ${ }^{1,3, b}$ \\ ${ }^{1}$ Physics Department and Biruni Observatory, College of Sciences, Shiraz University, Shiraz 71454, Iran \\ ${ }^{2}$ Physics Department, Faculty of Science, Shahid Chamran University of Ahvaz, Ahvaz 61357-43135, Iran \\ ${ }^{3}$ Research Institute for Astronomy and Astrophysics of Maragha (RIAAM), P. O. Box: 55134-441, Maragha, Iran
}

Received: 14 April 2018 / Accepted: 4 May 2018 / Published online: 17 May 2018

(C) The Author(s) 2018

\begin{abstract}
In this paper, we investigate the effects of nonlinear exponential electrodynamics as well as backreaction on the properties of one-dimensional $s$-wave holographic superconductors. We continue our study both analytically and numerically. In analytical study, we employ the SturmLiouville method while in numerical approach we perform the shooting method. We obtain a relation between the critical temperature and chemical potential analytically. Our results show a good agreement between analytical and numerical methods. We observe that the increase in the strength of both nonlinearity and backreaction parameters causes the formation of condensation in the black hole background harder and critical temperature lower. These results are consistent with those obtained for two dimensional $s$-wave holographic superconductors.
\end{abstract}

\section{Introduction}

The AdS/CFT duality provides a correspondence between a strongly coupled conformal field theory (CFT) in $d$ dimensions and a weakly coupled gravity theory in $(d+1)$ dimensional anti-de Sitter ( $A d S$ ) spacetime [1-3]. Since it is a duality between two theories with different dimensions, it is commonly called holography. The idea of holography has been employed in condensed matter physics to study various phenomena such as superconductors [4-8]. For describing the properties of low temperature superconductors, the BCS theory can work very well $[9,10]$. However, this theory fails to describe the mechanism of high temperature superconductor. In latter regime, the holography was suggested to study the properties of superconductors [11,12]. Hortnol et al. have represented the first model of holographic super-

\footnotetext{
a e-mail: mkzangeneh@scu.ac.ir

b e-mail: asheykhi@shirazu.ac.ir
}

conductors [11,12]. After that, holographic superconductors have attracted a lot of attention and investigated from different point of views [4,13-16].

BTZ black holes play a significant role in many of recent developments in string theory [17-19]. BTZ-like solutions are dual of $(1+1)$-dimensional holographic systems such as one-dimensional holographic superconductors. Distinctive features of normal and superconducting phases of onedimensional systems have been studied studied in [20]. The latter study was done in probe limit. Considering the effects of backreaction, the properties of one-dimensional holographic superconductors have been studied both numerically $[21,22]$ and analytically [23,24].

It is interesting to investigate the effect of nonlinear electrodynamic models on holographic systems including holographic superconductors [25-44]. Nonlinear models carry more information than the usual Maxwell case and also are considered as a possible way for avoiding the singularity of the point-like charged particle at the origin [45-49]. The oldest nonlinear electrodynamic model is Born-Infeld (BI) model. There are also two BI-like nonlinear electrodynamics namely logarithmic $[33,50,51]$ and exponential $[40,52,53]$ electrodynamics. It has been found that the exponential electrodynamics has stronger effect on the condensation than other models [54].

In the present work, we will study the one-dimensional holographic superconductors both analytically and numerically in the presence of exponential electrodynamics. To bring rich physics in holographic model, we consider the backreaction of the scalar and gauge fields on the metric background [55-61]. To perform the analytical study, we employ the Sturm-Liouville eigenvalue problem. We will study the effects of nonlinear exponential electrodynamics model as well as backreaction on critical temperature. We shall also use the numerical shooting method to investigate 
the features of our holographic superconductors and make comparison between analytical and numerical results.

This paper is organized as follows: In next section, we introduce the action and basic field equations governing $(1+1)$-dimensional holographic superconductors in the presence of exponential electrodynamics. In Sect. 3, we study the properties of holographic superconductors applying the analytical method based on Sturm-Liuoville eigenvalue problem. In Sect. 4, we study holographic superconductors numerically by employing the shooting method. We also compare our numerical and analytical results. Finally, in last section we will summarize our results.

\section{Holographic set-up}

To study a $(1+1)$-dimensional holographic superconductor, we consider a $(2+1)$-dimensional bulk action of AdS gravity coupled to a charged scalar field $\psi$

$$
\begin{aligned}
S= & \frac{1}{2 \kappa^{2}} \int d^{3} x \sqrt{-g}\left(R+\frac{2}{l^{2}}\right) \\
& +\int d^{3} x \sqrt{-g}\left[L(F)-|\nabla \psi-i q A \psi|^{2}-m^{2}|\psi|^{2}\right],
\end{aligned}
$$

where $g$ is the determinant of metric, $R$ is Ricci scalar, $l$ is the AdS radius, $A$ is electromagnetic potential and $F=F_{\mu \nu} F^{\mu \nu}$ in which $F_{\mu \nu}=\nabla_{[\mu} A_{\mu]}$. In action (1), $\kappa^{2}=8 \pi G_{3}$ where $G_{3}$ is the $(2+1)$-dimensional Newtonian constant and $m$ and $q$ represent the mass and charge of scalar field, respectively. $L(F)$ stands for the Lagrangian of electrodynamics model. $(1+1)$-dimensional holographic superconductors in the presence of linear Maxwell electrodynamics presented by $L(F)=-F / 4$ have been studied in $[21,62]$. The linear model is an idealization of reality. In principle, other powers of $F$ may play role. There are different nonlinear electrodynamics models which exhibit the electrodynamics interaction. In this paper, we suppose that electrodynamics interaction is governed by exponential nonlinear electrodynamics model [52]

$L(F)=\frac{1}{4 b}\left(\mathrm{e}^{-b F}-1\right)$,

where $b$ determines the nonlinearity. For small values of $b$, Lagrangian (2) recovers the linear Maxwell Lagrangian. The parameter $\kappa$ in (1) also determines the backreaction. When $\kappa$ goes to zero, we are in the probe limit, meaning that the gravity part of action (1) is stronger than the matter field part. Physically, this implies that the gauge and matter fields do not back react on the metric background. In superconducting language, implies that the Cooper pairs have negligible interaction with background system. In the presence of backreaction, the dual black hole solution may be given by the ansatz $d s^{2}=-f(r) \mathrm{e}^{-\chi(r)} d t^{2}+\frac{d r^{2}}{f(r)}+\frac{r^{2}}{l^{2}} d x^{2}$.

The Hawking temperature of above black hole solution is given by

$T=\frac{f^{\prime}\left(r_{+}\right) \mathrm{e}^{-\chi\left(r_{+}\right)}}{4 \pi}$,

where $r_{+}$is event horizon which could be obtained as the greatest root of $f(r)=0$. Varying the action (1) with respect to $\psi, A_{\nu}$ and $g_{\mu \nu}$, the field equations read, respectively,

$$
\begin{aligned}
0= & \left(\nabla_{\mu}-i q A_{\mu}\right)\left(\nabla^{\mu}-i q A^{\mu}\right) \psi-m^{2} \psi, \\
0= & \nabla^{\mu}\left(4 L_{F} F_{\mu \nu}\right) \\
& -i q\left[-\psi^{*}\left(\nabla_{\nu}-i q A_{\nu}\right) \psi+\psi\left(\nabla_{\nu}+i q A_{\nu}\right) \psi^{*}\right], \\
0= & \frac{1}{2 \kappa^{2}}\left[R_{\mu \nu}-g_{\mu \nu}\left(\frac{R}{2}+\frac{1}{l^{2}}\right)\right]+2 F_{a c} F_{b}{ }^{c} L_{F} \\
& -\frac{g_{\mu \nu}}{2}\left[L(F)-m^{2}|\psi|^{2}-|\nabla \psi-i q A \psi|^{2}\right] \\
& -\frac{1}{2}\left[\left(\nabla_{\mu} \psi-i q A_{\mu} \psi\right)\left(\nabla_{\nu} \psi^{*}+i q A_{\nu} \psi^{*}\right)+\mu \leftrightarrow v\right],
\end{aligned}
$$

where $L_{F}=\partial L / \partial F$. Adopting the ansatz $A_{\mu}=\phi(r) \delta_{\mu}^{0}$ and $\psi=\psi(r)$, field Eq. (5)-(7) lead to

$$
\begin{aligned}
0= & \psi^{\prime \prime}+\psi^{\prime}\left[\frac{1}{r}+\frac{f^{\prime}}{f}-\frac{\chi^{\prime}}{2}\right]+\psi\left[\frac{q^{2} \phi^{2} \mathrm{e}^{\chi}}{f^{2}}-\frac{m^{2}}{f}\right], \\
0= & \phi^{\prime \prime}+\phi^{\prime}\left(\frac{1}{r}+\frac{\chi^{\prime}}{2}\right) \\
& -\frac{2 q^{2} \psi^{2} \phi}{\mathrm{e}^{2 b \phi^{\prime 2} \mathrm{e} x}+2 b \phi^{\prime 2} \mathrm{e}^{\chi}\left(2 \phi^{\prime \prime}+\phi^{\prime} \chi^{\prime}\right),} \\
0= & f^{\prime}+\frac{\kappa^{2} r}{2 b}\left[1+\mathrm{e}^{2 b \phi^{\prime 2} \mathrm{e}^{\chi}}\left(4 b \phi^{\prime 2} \mathrm{e}^{\chi}-1\right)\right] \\
& +2 \kappa^{2} r\left[\frac{q^{2} \phi^{2} \psi^{2} \mathrm{e}^{\chi}}{f}+(m \psi)^{2}+\psi^{\prime 2} f\right]-\frac{2 r}{l^{2}}, \\
0= & \chi^{\prime}+4 \kappa^{2} r\left[\frac{q^{2} \phi^{2} \psi^{2} \mathrm{e}^{\chi}}{f^{2}}+\psi^{\prime 2}\right] .
\end{aligned}
$$

where the prime denotes the derivative with respect to $r$. Obviously, the above equations reduce to the corresponding equations in Ref. [21] when $b \rightarrow 0$ while in the absence of the backreaction $(\kappa \rightarrow 0)$, Eqs. (22) and (23) reduce to ones in Ref. [54]. By virtue of symmetries of field equations (22)-(25)

$$
\begin{aligned}
& q \rightarrow q / a, \phi \rightarrow a \phi, \psi \rightarrow a \psi, \\
& \kappa \rightarrow \kappa / a, b \rightarrow b / a^{2}, \\
& l \rightarrow a l, r \rightarrow a r, q \rightarrow q / a, \\
& m \rightarrow m / a, b \rightarrow a^{2} b,
\end{aligned}
$$


one can set $q=l=1$. In the following sections, we will study the superconding phase transition both analytically and numerically.

\section{Analytical study}

The behaviors of model functions governed by field equations (22)-(25) near the boundary $r \rightarrow \infty$ are $^{1}$

$$
\begin{aligned}
& \chi(r) \rightarrow 0, \quad f(r) \sim r^{2}, \\
& \phi(r) \sim \rho+\mu \ln (r), \quad \psi(r) \sim \frac{\psi_{-}}{r^{\Delta_{-}}}+\frac{\psi_{+}}{r^{\Delta_{+}}},
\end{aligned}
$$

where $\mu$ and $\rho$ are chemical potential and charge density of dual field theory and $\Delta_{ \pm}=1+\sqrt{1 \pm m^{2}}$. The superconducting phase transition is characterized by growing the expectation value of order parameter $\langle O\rangle$ as temperature decreases. In normal phase, $\langle O\rangle$ vanishes. According to holographic dictionary, the expectation value of order parameter $\langle O\rangle$ is dual to $\psi_{+}$or $\psi_{-}$while the other one can be considered as the source. Therefore, near the critical point $\left\langle O_{ \pm}\right\rangle$is small and one can define it as

$\epsilon \equiv\left\langle O_{i}\right\rangle$

where $i=+$ or - . Since $\epsilon$ is so small, we can expand the model functions as $[7,55,63,64]^{2}$

$f=f_{0}+\epsilon^{2} f_{2}+\epsilon^{4} f_{4}+\cdots$,

$\chi=\epsilon^{2} \chi_{2}+\epsilon^{4} \chi_{4}+\cdots$,

$\psi=\epsilon \psi_{1}+\epsilon^{3} \psi_{3}+\epsilon^{5} \psi_{5}+\cdots$,

$\phi=\phi_{0}+\epsilon^{2} \phi_{2}+\epsilon^{4} \phi_{4}+\cdots$.

Also we can expand the chemical potential as [7]

$\mu=\mu_{0}+\epsilon^{2} \delta \mu_{2}+\ldots$,

where $\delta \mu_{2}>0$. Thus, the order parameter as a function of chemical potential can be obtained as

$\epsilon \approx \frac{\left(\mu-\mu_{0}\right)^{1 / 2}}{\delta \mu_{2}}$.

When $\mu \rightarrow \mu_{0}$, phase transition occurs and the order parameter is zero at the critical value $\mu_{c}=\mu_{0}$. Above equation also indicates the critical exponent $\beta=1 / 2$ which is the same as the universal result from the mean field theory. Hereafter, we define the dimensionless coordinate $z=r_{+} / r$ instead of $r$, since it is easier to work with it. In terms of this new coordinate, $z=0$ and $z=1$ correspond to the boundary

\footnotetext{
1 Near the boundary, $\chi$ could be a constant but by using the symmetry of field equation $\mathrm{e}^{\chi} \rightarrow a^{2} \mathrm{e}^{\chi}, \phi \rightarrow \phi / a$, one can set it to zero there.

2 It is expected that when the sign of $\epsilon$ changes, the sign of scalar filed which leads to order parameter, changes too. So, the expansion powers of $\psi$ is considered odd. For other functions, even powers is used because they should not change when the sign of order parameter changes.
}

and horizon respectively. The field equations (22)-(25) can be rewritten in terms of $z$ as

$0=\psi^{\prime \prime}+\left[\frac{1}{z}+\frac{f^{\prime}}{f}-\frac{\chi^{\prime}}{2}\right] \psi^{\prime}+\frac{r_{+}^{2}}{z^{4}}\left[\frac{q^{2} \phi^{2} \mathrm{e}^{\chi}}{f^{2}}-\frac{m^{2}}{f}\right] \psi$,
$0=\phi^{\prime \prime}+\left(\frac{\chi^{\prime}}{2}+\frac{1}{z}\left[\frac{1+4 \Upsilon}{1+2 \Upsilon}\right]\right) \phi^{\prime}-\frac{2 q^{2} r_{+}^{2} \psi^{2}}{z^{4} f}\left(\frac{\mathrm{e}^{-\Upsilon}}{1+2 \Upsilon}\right) \phi$,

$0=f^{\prime}-\frac{\kappa^{2} r_{+}^{2}}{2 b z^{3}}\left[1+\mathrm{e}^{\Upsilon}(2 \Upsilon-1)\right]$

$-\frac{2 \kappa^{2} r_{+}^{2}}{z^{3}}\left[\frac{q^{2} \phi^{2} \psi^{2} \mathrm{e}^{\chi}}{f}+m^{2} \psi^{2}\right]+\frac{2 r_{+}^{2}}{z^{3}}$,

$0=\chi^{\prime}-\frac{4 \kappa^{2} r_{+}^{2}}{z^{3}}\left[\frac{q^{2} \phi^{2} \psi^{2} \mathrm{e}^{\chi}}{f^{2}}+\frac{z^{4} \psi^{\prime 2}}{r_{+}^{2}}\right]$,

where $\Upsilon=2 b z^{4} \mathrm{e}^{\chi} \phi^{\prime 2} / r_{+}{ }^{2}$. The field equation of $\phi$ (Eq. (23)) at zeroth order with respect to $\epsilon$ reduces to

$\phi^{\prime \prime}(z)+\frac{\phi^{\prime}(z)\left(r_{+}^{2}+8 b z^{4} \phi^{2}(z)\right)}{z\left(r_{+}^{2}+4 b z^{4} \phi^{\prime 2}(z)\right)}=0$.

The solution of above equation reads

$\phi(z)=\int_{1}^{z} \frac{r+\sqrt{L_{W}\left(\frac{4 b z^{2} C_{0}^{2}}{r_{+}{ }^{2}}\right)}}{2 z^{2} \sqrt{b}} d z$

where $C_{0}$ is an integration constant and $L_{W}(x)=$ Lambert$W(x)$ is the Lambert function which satisfies [65]

$L_{W}(x) \mathrm{e}^{L_{W}(x)}=x$,

and can be expanded as

$L_{W}(x)=x-x^{2}+\frac{3}{2} x^{3}-\frac{8}{3} x^{4}$.

Expanding Eq. (27) for small $b$ and keeping the terms up to first order of $b$ we find

$\phi(z)=C_{1}+C_{0} \ln (z)+\frac{C_{0}^{3}}{r_{+}^{2}}\left(1-z^{2}\right) b+O\left(b^{2}\right)$.

Comparing the above equation with Eq. (14), we find $C_{0}=$ $-\mu$. Also, $C_{1}=0$, since at the horizon $\phi\left(r_{+}\right)=0$. Inserting $C_{0}$ into Eq. (30) we have

$\phi_{0}(z)=\lambda r_{+}\left[-\ln (z)+\lambda^{2}\left(z^{2}-1\right) b\right], \quad b \lambda^{2}<1$,

where $\lambda=\mu / r_{+}$. Substituting $\phi(z)$ into the field equation (24), we find the metric function at zeroth order with respect to $\epsilon, f_{0}(z)=r_{+}^{2} g(z)$ where

$g(z)=\left(\frac{1}{z^{2}}-1+\kappa^{2} \lambda^{2} \ln (z)+\frac{1}{2} \kappa^{2} \lambda^{4} b\left(1-z^{2}\right)\right)$.

Note that at the horizon $f_{0}(1)=0$. The asymptotic behavior of scalar field $\psi$ near the boundary $(z=0)$ is given by Eq. (14). In order to match this behavior near the boundary, we introduce a trial function $F(z)$ as $\psi(z)=$ 
Table 1 Analytical results of $T_{c} / \mu$ for different values of $\kappa$ and $b$

\begin{tabular}{llllll}
\hline$b$ & $\kappa^{2}=0$ & $\kappa^{2}=0.005$ & $\kappa^{2}=0.01$ & $\kappa^{2}=0.015$ & $\kappa^{2}=0.02$ \\
\hline 0 & 0.0429 & 0.0393 & 0.0379 & 0.0369 & 0.0359 \\
0.01 & 0.0342 & 0.0321 & 0.0311 & 0.0301 & 0.0288 \\
0.02 & 0.0274 & 0.0231 & 0.0216 & 0.0208 & 0.0199 \\
\hline
\end{tabular}

$\left\langle O_{i}\right\rangle\left(z / r_{+}\right)^{\Delta_{i}} F(z)$ which satisfies the boundary condition $F(0)=1$ and $F^{\prime}(0)=0$. Inserting the functions obtained above and the trial function $F(z)$ into Eq. (22), one receives

$$
\begin{aligned}
& F^{\prime \prime}(z)+F^{\prime}(z)\left[\frac{2 \Delta+1}{z}+\frac{g^{\prime}(z)}{g(z)}\right] \\
& +\left[-\frac{m^{2}}{g(z) z^{4}}+\frac{\Delta^{2}}{z^{2}}+\frac{\Delta g^{\prime}(z)}{z g(z)}\right] F(z)+ \\
& +\frac{q^{2} \lambda^{2} F(z)}{g(z)^{2}}\left[\frac{\ln (z)^{2}}{z^{4}}-\frac{2 \lambda^{2} b \ln (z)}{z^{2}}+\frac{2 \lambda^{2} b \ln (z)}{z^{4}}\right]=0 .
\end{aligned}
$$

It is a matter of calculations to show that the above equation satisfies the following second order Sturm-Liouville equation [66]

$$
\left[T(z) F^{\prime}(z)\right]^{\prime}-Q(z) F(z)+\lambda^{2} P(z) F(z)=0,
$$

where

$$
\begin{aligned}
& T(z)=g(z) z^{2 \Delta+1}, \\
& P(z)=\frac{q^{2} T(z)}{g^{2}(z)}\left[\frac{\ln (z)^{2}}{z^{4}}-\frac{2 \lambda^{2} b \ln (z)}{z^{2}}+\frac{2 \lambda^{2} b \ln (z)}{z^{4}}\right], \\
& Q(z)=-T(z)\left[-\frac{m^{2}}{g(z) z^{4}}+\frac{\Delta^{2}}{z^{2}}+\frac{\Delta g^{\prime}(z)}{z g(z)}\right] .
\end{aligned}
$$

Considering the trial function as $F(z)=1-\alpha z^{2}$ and using the Sturm-Liouville eigenvalues problem, the eigenvalues of Eq. (34) can be obtained by minimizing

$\lambda^{2}=\frac{\int_{0}^{1} d z T(z)\left[F^{\prime 2}(z)+Q(z) F^{2}(z)\right]}{\int_{0}^{1} d z \cdot T(z) P(z) F^{2}(z)}$,

with respect to $\alpha$ [67]. Here, we use a perturbative expansion $b \lambda^{2}$ up to the first order of $b$

$b \lambda^{2}=b\left(\left.\lambda^{2}\right|_{b=0}\right)+O\left(b^{2}\right)$.

For backreaction parameter, we use iteration method and take

$\kappa_{n}=n \Delta \kappa, \quad n=0,1,2,3, \ldots$,

where $\Delta \kappa=\kappa_{n+1}-\kappa_{n}$. Here we chose $\Delta \kappa=0.005$. So, the effect of the nonlinear corrections on the backreaction term can be obtained as

$\kappa^{2} \lambda^{2}=\kappa_{n}{ }^{2} \lambda^{2}=\kappa_{n}{ }^{2}\left(\left.\lambda^{2}\right|_{\kappa_{n-1}}\right)+O\left[(\Delta \kappa)^{4}\right]$.
By taking $\kappa_{-1}=0$ and $\left.\lambda^{2}\right|_{\kappa_{-1}}=0$, the minimum eigenvalue of Eq. (38) can be calculated. To calculate the critical temperature, we obtain the latter value by variation of Eq. (38) with respect to $\alpha$ where the other parameters such as $b, \kappa, m, q, \ldots$ are fixed. Using the definition of $T$ (Eq. (4)), the critical temperature is given by $T_{c}=f^{\prime}\left(r_{+}\right) / 4 \pi$ where $^{3}$

$f^{\prime}\left(r_{+}\right)=2 r_{+}-\frac{\kappa^{2} r_{+}}{2 b}\left[1+\mathrm{e}^{2 b \phi_{0}^{\prime 2}\left(r_{+}\right)}\left(4 b \phi_{0}^{\prime 2}\left(r_{+}\right)-1\right)\right]$,

and $r_{+}=\mu / \lambda$ so we have

$T_{c}=\frac{1}{4 \pi} \frac{\mu}{\lambda}\left[2-\kappa_{n}{ }^{2}\left(\left.\lambda^{2}\right|_{\kappa_{n-1}}\right)+3 b \kappa_{n}{ }^{2}\left(\left.\lambda^{4}\right|_{\kappa_{n-1}, b=0}\right)\right]$.

As an example, for $b=0.01$ and $\kappa=0$ the Eq. (38) reduce to

$\lambda^{2}=\frac{0.667 \alpha^{2}-1.333 \alpha+1}{0.0276-0.0165 \alpha+0.0035 \alpha^{2}}$,

which has a minimum $\lambda_{\min }=21.6337$, with respect to $\alpha$, at $\alpha=0.8258$ and thus according to Eq. (43) we achieve $T_{c}=0.03421 \mu$. We employ the iteration method to obtain the critical temperature for different values of $\kappa$ and $b$.

In Table 1, we present our results. This table shows that by increasing nonlinear parameter $(b)$, the value of $T_{c}$ decreases. As it can be understood from this table, for a fixed value of $b$, with increasing the backreaction parameter $\kappa$, the value of the critical temperature decreases. We also reproduce the results of the linear Maxwell case without backreaction (i.e. $b \rightarrow 0$ and $\kappa \rightarrow 0$ ) presented in [23].

Table 2 Numerical results of $T_{c} / \mu$ for different values of $\kappa$ and $b$

\begin{tabular}{llllll}
\hline$b$ & $\kappa^{2}=0$ & $\kappa^{2}=0.05$ & $\kappa^{2}=0.1$ & $\kappa^{2}=0.15$ & $\kappa^{2}=0.2$ \\
\hline 0 & 0.046 & 0.0368 & 0.0295 & 0.0236 & 0.0189 \\
0.01 & 0.0415 & 0.033 & 0.0262 & 0.0209 & 0.0166 \\
0.04 & 0.0325 & 0.0252 & 0.0197 & 0.0154 & 0.0121 \\
0.09 & 0.0236 & 0.0176 & 0.0133 & 0.0107 & 0.0078 \\
\hline
\end{tabular}

\footnotetext{
3 Note that at zeroth order with respect to $\epsilon, \chi$ is zero. So, $\mathrm{e}^{\chi}$ in temperature formula disappears.
} 


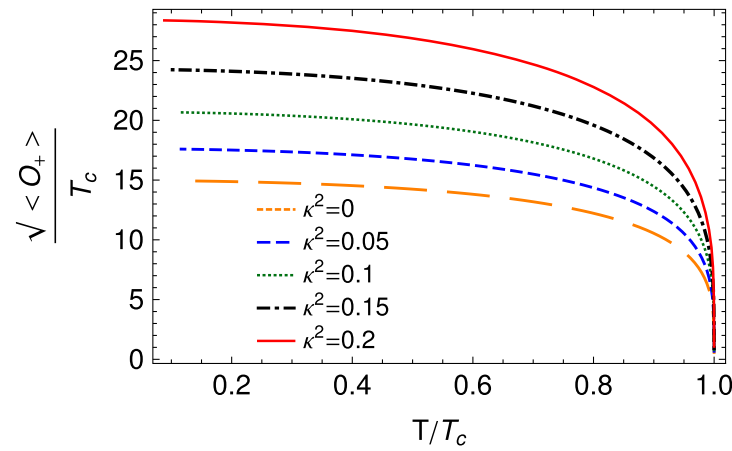

(a) $b=0$

Fig. 1 The behavior of order parameter versus temperature for $m^{2}=0$

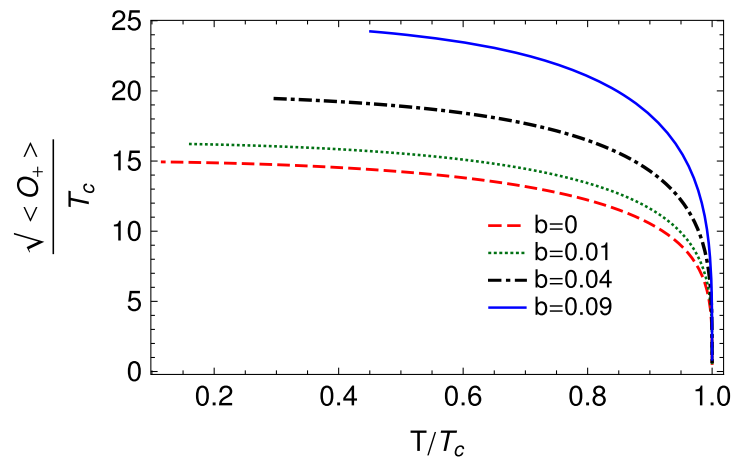

(a) $\kappa^{2}=0$

Fig. 2 The behavior of order parameter versus temperature for $m^{2}=0$

\section{Numerical study}

In this section, we employ the shooting method [4] to numerically investigate the superconducting phase transition. Besides setting $q$ and $l$ to unity, we also set $r_{+}=1$ in the numerical calculation which may be justified by virtue of the field equation symmetry [21]

$r \rightarrow a r, f \rightarrow a^{2} f, \phi \rightarrow a \phi$,

First, we expand Eqs. (22)-(25) near black hole horizon $(z=$ 1)

$$
\begin{aligned}
\psi & \approx \psi(1)+\psi^{\prime}(1)(1-z)+\frac{\psi^{\prime \prime}}{2}(1-z)^{2}+\cdots, \\
\phi & \approx \phi^{\prime}(1)(1-z)+\frac{\phi^{\prime \prime}}{2}(1-z)^{2}+\cdots, \\
f & \approx f^{\prime}(1)(1-z)+\frac{f^{\prime \prime}}{2}(1-z)^{2}+\cdots, \\
\chi & \approx \chi(1)+\chi^{\prime}(1)(1-z)+\frac{\chi^{\prime \prime}}{2}(1-z)^{2}+\cdots .
\end{aligned}
$$

In above equations, we have imposed $f(1)=\phi(1)=0 .{ }^{4}$ In our numerical process, we will find $\psi(1), \phi^{\prime}(1)$ and $\chi(1)$

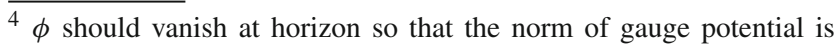
regular there.
}

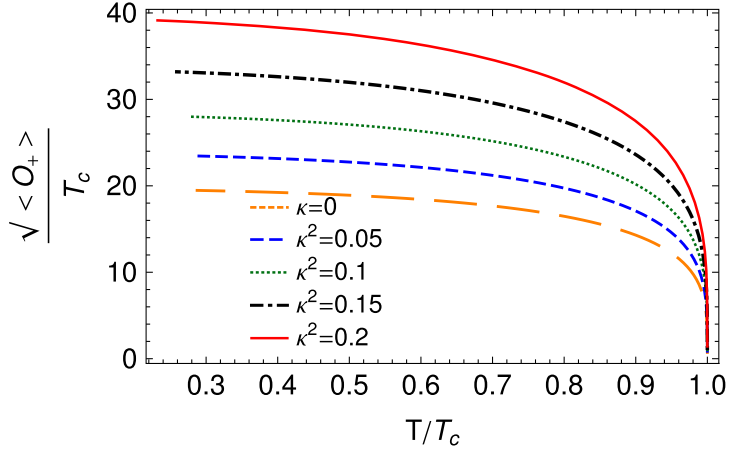

(b) $b=0.04$

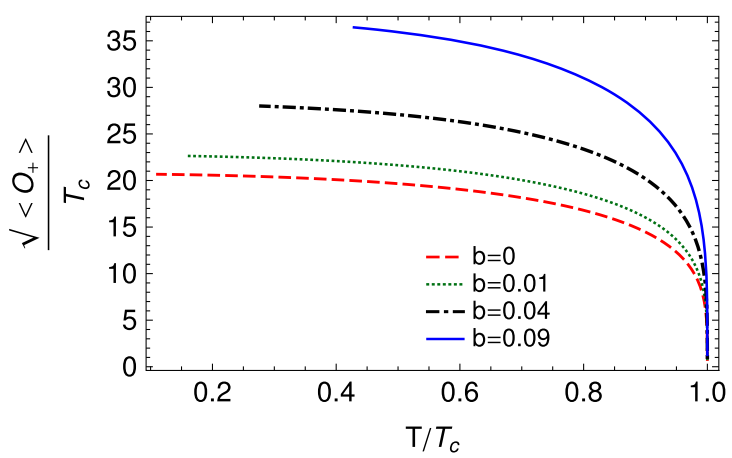

(b) $\kappa^{2}=0.1$

such that the desired values for boundary parameters in Eq. (14) are attained. At boundary, one can set either $\psi_{-}$or $\psi_{+}$ to zero as source and find the value of the other one as the expectation value of order parameter $\langle O\rangle$. We will focus on $m^{2}=0$ case for our numerical calculations. For this case, the behavior of $\psi$ near boundary is (see Eq. (14))

$\psi(z) \approx \psi_{-}+\psi_{+} z^{2}$

We consider $\psi_{+}$as holographic dual to the order parameter $\left\langle\mathrm{O}_{+}\right\rangle$at the boundary field theory.

In Table 2, our numerical results for critical temperature with different values of backreaction parameter $\kappa$ and nonlinear parameter $b$ are presented. In the Maxwell limit $(b \rightarrow 0)$, our numerical results reproduce the ones of [21]. It can be seen that in the absence of nonlinearity effect, the critical temperature decreases as $\kappa$ increases [21]. In the presence of nonlinearity parameter i.e. for any nonvanishing value of $b$, it can be found that as $\kappa$ enhances, the critical temperature $T_{c}$ decreases. Similar behavior can be found for different values of $b$ when $\kappa$ is fixed. As the nonlinear parameter $b$ becomes larger, the critical temperature decreases i.e. the condensation process becomes harder. This behavior have been reported previously in [68] for $(2+1)$-dimensional holographic superconductors too. Figures 1 and 2 confirm 
Table 3 Analytical and numerical results of $T_{c} / \mu$ for different values of $\kappa$ and $b$

\begin{tabular}{|c|c|c|c|c|c|c|c|c|c|c|}
\hline \multirow[t]{2}{*}{$b$} & \multicolumn{2}{|l|}{$\kappa^{2}=0$} & \multicolumn{2}{|c|}{$\kappa^{2}=0.005$} & \multicolumn{2}{|c|}{$\kappa^{2}=0.01$} & \multicolumn{2}{|c|}{$\kappa^{2}=0.015$} & \multicolumn{2}{|c|}{$\kappa^{2}=0.02$} \\
\hline & $T_{c}(\mathrm{An})$ & $T_{c}(\mathrm{Nu})$ & $T_{c}(\mathrm{An})$ & $T_{c}(\mathrm{Nu})$ & $T_{c}(\mathrm{An})$ & $T_{c}(\mathrm{Nu})$ & $T_{c}(\mathrm{An})$ & $T_{c}(\mathrm{Nu})$ & $T_{c}(\mathrm{An})$ & $T_{c}(\mathrm{Nu})$ \\
\hline 0 & 0.0429 & 0.046 & 0.0393 & 0.0449 & 0.0379 & 0.0439 & 0.0369 & 0.0430 & 0.0359 & 0.0409 \\
\hline 0.01 & 0.0342 & 0.0415 & 0.0321 & 0.0406 & 0.0311 & 0.0396 & 0.0301 & 0.0387 & 0.0288 & 0.0378 \\
\hline 0.02 & 0.0274 & 0.0380 & 0.0231 & 0.0371 & 0.0216 & 0.0362 & 0.0208 & 0.0353 & 0.0199 & 0.0345 \\
\hline
\end{tabular}

above results. As it can be seen from Fig. 1, the scalar hair forms harder as $\kappa$ increases i.e. the gap in graph of $\left\langle O_{+}\right\rangle$ becomes larger. The latter means that the condensation of the operator $\left\langle O_{+}\right\rangle$starts at larger values for stronger values of backreaction parameter. It shows that the scalar hair can be formed more difficult when the backreaction is stronger. Figure 2 shows the same effect for nonlinearity parameter $b$. We compare the analytical and numerical results in Table 3. Table 3 shows that there is a good agreement between analytical and numerical results for small values of $\kappa$ and $b$. For larger values of these parameters, analytical and numerical results separate more from each other.

\section{Conclusion}

In this work, we have studied the properties of onedimensional holographic superconductor in the presence of nonlinear exponential electrodynamics. We have also considered the backreaction effect of scalar and gauge fields on the background metric. We have performed both analytical and numerical methods for studying our superconductors. To investigate the problem analytically, we have used the Sturm-Lioville while our numerical study was based on shooting method. It was shown that the enhancement in both nonlinearity of electrodynamics model as well as the backreaction causes the superconducting phase more difficult to be appeared. This result is reflected in two ways from our data. From one side, we observed that the increasement in the effects of nonlinearity and backreaction makes the critical temperature of superconductor lower. From another side, for larger values of nonlinear and backreaction parameters, the gap in condensation parameter is larger which in turn exhibits that the condensation is formed harder. We have also observed that, for small values of backreaction parameter $\kappa$ and nonlinear parameter $b$, the analytic results are in a good agreement with numerical ones whereas for larger values they separate more from each other.

Finally, we would like to stress that in this work, we have only studied the basic properties of one-dimensional backreacting holographic $s$-wave superconductors in the presence of exponential nonlinear electrodynamics. It is also interesting to investigate other characteristics of these systems such as the behaviour of real and imaginary parts of conductivity or optical features. One may also consider $(1+1)$ dimensional $p$-wave and $d$-wave holographic superconductors in the background of BTZ black holes and disclose the effects of nonlinearity as well as backreaction on the the phase transition and conductivity of these models. These issue are now under investigations and the result will be appeared soon.

Acknowledgements MKZ would like to thank Shahid Chamran University of Ahvaz, Iran for supporting this work. AS thanks the research council of Shiraz University. The work of MKZ has been supported financially by Research Institute for Astronomy and Astrophysics of Maragha (RIAAM) under research Project no. 1/5237-55.

Open Access This article is distributed under the terms of the Creative Commons Attribution 4.0 International License (http://creativecomm ons.org/licenses/by/4.0/), which permits unrestricted use, distribution, and reproduction in any medium, provided you give appropriate credit to the original author(s) and the source, provide a link to the Creative Commons license, and indicate if changes were made. Funded by SCOAP 3 .

\section{References}

1. J.M. Maldacena, The large-N limit of superconformal field theories and supergravity. Adv. Theor. Math. Phys. 2, 231 (1998). arXiv:hep-th/9711200

2. S.S. Gubser, I.R. Klebanov, A.M. Polyakov, Gauge theory correlators from non-critical string theory. Phys. Lett. B 428, 105 (1998). arXiv:hep-th/9802109

3. E. Witten, Anti-de Sitter space and holography. Adv. Theor. Math. Phys. 2, 253 (1998). arXiv:hep-th/9802150

4. S.A. Hartnoll, Lectures on holographic methods for condensed matter physics. Class. Quant. Grav. 26, 224002 (2009). arXiv:0903.3246

5. C.P. Herzog, Lectures on holographic superfluidity and superconductivity. J. Phys. A 42, 343001 (2009). arXiv:0904.1975

6. J. McGreevy, Holographic duality with a view toward manybody physics. Adv. High Energy Phys. 2010, 723105 (2010). arXiv:0909.0518

7. C.P. Herzog, Analytic holographic superconductor. Phys. Rev. D 81, 126009 (2010). arXiv: 1003.3278

8. S.S. Gubser, Breaking an Abelian gauge symmetry near a black hole horizon. Phys. Rev. D 78, 065034 (2008). arXiv:0801.2977

9. J. Bardeen, L.N. Cooper, J.R. Schrieffer, Microscopic Theory of Superconductivity. Phys. Rev. 106, 162 (1957)

10. J. Bardeen, L.N. Cooper, J.R. Schrieffer, Theory of superconductivity. Phys. Rev. 108, 1175 (1957) 
11. S.A. Hartnoll, C.P. Herzog, G.T. Horowitz, Building a holographic superconductor. Phys. Rev. Lett. 101, 031601 (2008). arXiv:0803.3295

12. S.A. Hartnoll, C.P. Herzog, G.T. Horowitz, Holographic superconductors. JHEP 12, 015 (2008). arXiv:0810.1563

13. G.T. Horowitz, M.M. Roberts, Holographic superconductors with various condensates. Phys. Rev. D 78, 126008 (2008). arXiv:0810.1077

14. S. Franco, A. Garcia-Garcia, D. Rodriguez-Gomez, A general class of holographic superconductors. JHEP 04, 092 (2010). arXiv:0906.1214

15. Q.Y. Pan, B. Wang, General holographic superconductor models with Gauss-Bonnet corrections. Phys. Lett. B 693, 159 (2010). arXiv: 1005.4743

16. X.H. Ge, B. Wang, S.F. Wu, G.H. Yang, Analytical study on holographic superconductors in external magnetic field. JHEP 08, 108 (2010). arXiv:1002.4901

17. K. Skenderis, Black holes and branes in string theory. Lect. Notes Phys. 541, 325 (2000). arXiv:hep-th/9901050

18. S. Hyun, U-duality between three and higher dimensional black holes. J. Kor. Phys. Soc. 33, S532 (1998). arXiv:hep-th/9704005

19. A. Strominger, Black hole entropy from near horizon microstates. JHEP 9802, 009 (1998). arXiv:hep-th/9712251

20. J. Ren, One-dimensional holographic superconductor from $A d S_{3} / C F T_{2}$ correspondence. JHEP 1011, 055 (2010). arXiv: 1008.3904

21. Y. Liu, Q. Pan, B. Wang, Holographic superconductor developed in BTZ black hole background with backreactions. Phys. Lett. B 702, 94 (2011). arXiv: 1106.4353

22. M. Kord Zangeneh, Y.C. Ong, B. Wang, Entanglement entropy and complexity for one-dimensional holographic superconductors. Phys. Lett. B 771, 235 (2017). arXiv:1704.00557

23. R. Li, Note on analytical studies of one dimensional holographic superconductors. Mod. Phys. Lett. A 27, 1250001 (2012)

24. D. Momeni, M. Raza, M.R. Setare, R. Myrzakulov, Analytical holographic superconductor with backreaction using $A d S_{3} / C F T_{2}$. Int. J. Theor. Phys. 52, 2773 (2013). arXiv:1305.5163

25. Y. Liu, Y. Peng, B., Wang: Gauss-Bonnet holographic superconductors in Born-Infeld electrodynamics with backreactions. arXiv: 1202.3586

26. Y. Liu, Y. Gong, B. Wang, Non-equilibrium condensation process in holographic superconductor with nonlinear electrodynamics. JHEP 02, 116 (2016). arXiv: 1505.03603

27. D. Roychowdhury, Effect of external magnetic field on holographic superconductors in presence of non-linear corrections. Phys. Rev. D 86, 106009 (2012). arXiv: 1211.0904

28. J. Jing, S. Chen, Holographic superconductors in the Born-Infeld electrodynamics. Phys. Lett. B 686, 68 (2010). arXiv:1001.4227

29. R. Banerjee, S. Gangopadhyay, D. Roychowdhury, A. Lala, Holographic s-wave condensate with nonlinear electrodynamics: a nontrivial boundary value problem. Phys. Rev. D 87, 104001 (2013). arXiv: 1208.5902

30. J. Jing, Q. Pan, S. Chen, Holographic superconductors with PowerMaxwell field. JHEP 11, 045 (2011). arXiv:1106.5181

31. Y. Liu, B. Wang, Perturbations around the AdS Born-Infeld black holes. Phys. Rev. D 85, 046011 (2012). arXiv:1111.6729

32. A. Sheykhi, H.R. Salahi, A. Montakhab, Analytical and numerical study of Gauss-Bonnet holographic superconductors with PowerMaxwell field. JHEP 04, 058 (2016). arXiv:1603.00075

33. J. Jing, Q. Pan, S. Chen, Holographic superconductor/insulator transition with logarithmic electromagnetic field in Gausse Bonnet gravity. Phys. Lett. B 716, 385 (2012). arXiv:1209.0893

34. C. Lai, Q. Pan, J. Jing, Y. Wang, On analytical study of holographic superconductors with Born-Infeld electrodynamics. Phys. Lett. B 749, 437 (2015). arXiv:1508.05926
35. A. Sheykhi, F. Shaker, Analytical study of holographic superconductor in Born-Infeld electrodynamics with backreaction. Phys. Lett. B 754, 281 (2016). arXiv:1601.04035

36. S. Gangopadhyay, Holographic superconductors in Born-Infeld electrodynamics and external magnetic field. Mod. Phys. Lett. A 29, 1450088 (2014). arXiv:1311.4416

37. A. Sheykhi, A. Ghazanfari, A. Dehyadegari, Holographic conductivity of holographic superconductors with higher order corrections. Eur. Phys. J. C 78, 159 (2018). arXiv:1712.04331

38. A. Sheykhi, D. Hashemi Asl, A. Dehyadegari, Conductivity of higher dimensional holographic superconductors with nonlinear electrodynamics. Phys. Lett. B 781, 139 (2018). arXiv:1803.05724

39. M. Kord Zangeneh, S. S. Hashemi, A. Dehyadegari, A. Sheykhi, B. Wang, Optical properties of Born-Infeld-dilaton-Lifshitz, holographic superconductors. arXiv: 1710.10162

40. Z. Sherkatghanad, B. Mirza, F. Lalehgani Dezaki, Exponential nonlinear electrodynamics and backreaction effects on holographic superconductor in the lifshitz black hole background. Int. J. Mod. Phys. D 27, 1750175 (2017). arXiv:1708.04289

41. A. Dehyadegari, M. Kord Zangeneh, A. Sheykhi, Holographic conductivity in the massive gravity with power-law Maxwell field. Phys. Lett. B 773, 344 (2017). arXiv:1703.00975

42. A. Dehyadegari, A. Sheykhi, M. Kord Zangeneh, Holographic conductivity for logarithmic charged Dilaton-Lifshitz solutions. Phys. Lett. B 758, 226 (2016). arXiv: 1602.08476

43. M. Kord Zangeneh, A. Dehyadegari, A. Sheykhi, M.H. Dehghani, Thermodynamics and gauge/gravity duality for Lifshitz black holes in the presence of exponential electrodynamics. JHEP 1603, 037 (2016). arXiv: 1601.04732

44. A. Sheykhi, F. Shamsi, S. Davatolhagh, The upper critical magnetic field of holographic superconductor with conformally invariant Power-Maxwell electrodynamics. Can. J. Phys. 95, 450 (2017). arXiv: 1609.05040

45. M. Born, L. Infeld, Foundations of the new field theory. Proc. R. Soc. A 144, 425 (1934)

46. B. Hoffmann, Gravitational and electromagnetic mass in the BornInfeld electrodynamics. Phys. Rev. 47, 877 (1935)

47. W. Heisenberg, H.Z. Euler, Consequences of Dirac theory of the positron. Physics 98, 714 (1936). arXiv:physics/0605038 [physics.hist-ph]

48. H.P. de Oliveira, Non-linear charged black holes. Class. Quant. Grav. 11, 1469 (1994)

49. G.W. Gibbons, D.A. Rasheed, Electric-magnetic duality rotations in non-linear electrodynamics. Nucl. Phys. B 454, 185 (1995). arXiv:hep-th/9506035

50. H.H. Soleng, Charged black points in general relativity coupled to the logarithmic U(1) gauge theory. Phys. Rev. D 52, 6176 (1995). arXiv:hep-th/9509033

51. A. Sheykhi, M.H. Dehghani, M. Kord Zangeneh, Thermodynamics of charged rotating dilaton black branes coupled to logarithmic nonlinear electrodynamics. Adv. High Energy Phys. 2016, 3265968 (2016). arXiv: 1604.05300

52. S.H. Hendi, Asymptotic charged BTZ black hole solutions. JHEP 1203, 065 (2012). arXiv: 1405.4941

53. S.H. Hendi, A. Sheykhi, Charged rotating black string in gravitating nonlinear electromagnetic fields. Phys. Rev. D 88, 044044 (2013). arXiv: 1405.6998

54. Z. Zhao, Q. Pan, S. Chen, J. Jing, Notes on holographic superconductor models with the nonlinear electrodynamics. Nucl. Phys. 871, 98 (2013). arXiv: 1212.6693

55. Q. Pan, J. Jing, B. Wang, S. Chen, Analytical study on holographic superconductors with backreactions. JHEP 06, 087 (2012). arXiv: 1205.3543

56. S.S. Gubser, A. Nellore, Low-temperature behavior of the Abelian Higgs model in anti-de Sitter space. JHEP 04, 008 (2009). arXiv:0810.4554 
57. Y. Brihaye, B. Hartmann, Holographic superconductors in $3+1$ dimensions away from the probe limit. Phys. Rev. D 81, 126008 (2010). arXiv: 1003.5130

58. G.T. Horowitz, B. Way, Complete phase diagrams for a holographic superconductor/insulator system. JHEP 11, 011 (2010). arXiv: 1007.3714

59. A. Akhavan, M. Alishahiha, P-wave holographic insulator/superconductor phase transition. Phys. Rev. D 83, 086003 (2011). arXiv:1011.6158

60. S. Gangopadhyay, Analytic study of properties of holographic superconductors away from the probe limit. Phys. Lett. B 724, 176 (2013). arXiv: 1302.1288

61. T. Konstandin, G. Nardini, M. Quiros, Gravitational Backreaction effects on the holographic phase transition. Phys. Rev. D 82, 083513 (2010). arXiv:1007.1468

62. J. Ren, One-dimensional holographic superconductor from $A d S_{3} / C F T_{2}$ correspondence. JHEP 1011, 055 (2010). arXiv: 1008.3904
63. S. Kanno, A note on Gauss-Bonnet holographic superconductors. Class. Quant. Grav. 28, 127001 (2011). arXiv:1103.5022

64. G.E. Xian-Hui, L.E.N.G. Hong-Qiang, Analytical calculation on critical magnetic field in holographic superconductors with backreaction. Prog. Theor. Phys. 128, 1211 (2012). arXiv:1105.4333

65. M. Abramowitz, I.A. Stegun, Handbook of mathematical functions (Dover, New York, 1972)

66. S. Gangopadhyay, D. Roychowdhury, Analytic study of properties of holographic superconductors in Born-Infeld electrodynamics. JHEP 05, 002 (2012). arXiv: 1201.6520

67. G. Siopsis, J. Therrien, Analytical calculation of properties of holographic superconductors. JHEP 1005, 013 (2010). arXiv: 1003.4275

68. A. Sheykhi, F. Shaker, Effects of backreaction and exponential nonlinear electrodynamics on the holographic superconductors. Int. J. M. Phys. D 26, 1750050 (2017). arXiv:1606.04364 [gr-qc] 